\title{
STRUCTURE DU PEUPLEMENT PISCICOLE À L'ÉCHELLE D'UN GRAND BASSIN EUROPÉEN : ORIGANISATION LONGITUDINALE, INFLUENCE DE LA PENTE ET TENDANCES RÉGIONALES.
}

\author{
T. CHANGEUX* \\ Laboratoire d'Écologie des Systèmes Fluviaux, DESMID, URA CNRS 1974, \\ 1 rue Parmentier, 13200 Arles, France.
}

\section{RÉSUMÉ}

A large échelle, les peuplements de poissons européens sont soumis à trois influences majeures. D'abord, le gradient amont-aval des habitats engendré par le mouvement de l'eau organise le peuplement dans la dimension longitudinale. Ensuite, des discontinuités apparaissent dans cette organisation sous l'influence des variations de pente. Enfin, les différentes régions écologiques agissent également sur la distribution des espèces. Le bassin du Rhône peut servir de modèle pour l'étude de ces trois influences car il rassemble, sur une petite étendue, les contrastes régionaux qui dominent l'Europe.

Dans la dimension longitudinale, la pente et la distance à la source interagissent sur les communautés des cours supérieurs et médians, une variation de pente donnée n'ayant pas le même effet suivant la distance à la source.

Une part importante de la variabilité des communautés reste expliquée par des distinctions sectorielles, montrant l'influence de conditions locales qui ne sont pas prises en compte dans la dimension longitudinale et la pente. Ces variations s'inscrivent en conformité avec trois régions ichtyogéographiques déjà identifiées sur le bassin. Elles correspondent aux influences climatiques alpine, méridionale et océanique à tendance continentale.

Mots-clés : peuplement européen de poissons, gradient longitudinal, régions écologiques, bassin du Rhône.

\section{FISH COMMUNITY STRUCTURE AT THE SCALE OF A LARGE EUROPEAN BASIN : LONGITUDINAL ORGANISATION, SLOPE INFLUENCE AND REGIONAL TENDENCIES.}

\begin{abstract}
On a broad scale, the European fish community is under three major influences. First, the upstream-downstream gradient in habitat generated by water flow organizes the community in its longitudinal dimension. Then, discontinuities appear in this organization under the influence of slope variations. Finally, the different ecological regions have an effect on the distribution of the species. The Rhone Basin can be used as a model for the study of these three influences because it contains, over a small area, the regional heterogeneities which dominate in Europe.
\end{abstract}

In the longitudinal dimension, slope and distance from the source interact on the fish communities of the upper and median courses. A variation in slope has different effects according to the distances from the source.

An great part of the variation in communities is also explained by differences in geographic zones, thus showing the influence of local conditions which are not taken into

\footnotetext{
*Adresse actuelle : Conseil Supérieur de la Pêche, Direction Générale, 134 avenue de Malakoff, 75116 Paris, France.
} 
account by the longitudinal dimension and the slope. These regional variations fall within the three ichthyogeographic regions previously identified within the basin: the Alpine, southern and oceanic climatic subdivisions, with a continental modulation.

Key-words : European fish community, longitudinal gradient, ecological regions, Rhone basin.

\section{INTRODUCTION}

La relation habitat-poisson peut être considérée à différentes échelles. Les bassins fluviaux qui rassemblent les cours d'eau par unité de drainage, depuis la source jusqu'à la mer, sont les plus grandes unités géographiques de connexion pour les systèmes d'eaux courantes. Ils représentent, à ce titre, des unités de peuplement pour les poissons d'eau douce. A cette échelle, trois influences majeures peuvent agir dans la relation habitat-poisson :

1 - D'abord, le mouvement de l'eau au sein des unités morphologiques est à l'origine d'un gradient longitudinal des habitats. A l'équilibre, la taille et le débit des rivières augmentent avec la distance à la source tandis que l'altitude et la pente diminuent. A ce gradient théorique du milieu physique, correspond une organisation longitudinale du peuplement piscicole qui varie avec les caractéristiques propres à chaque cours d'eau.

2 - Dans cette relation habitat-poisson, où tous les paramètres sont fortement liés les uns aux autres, il est difficile de savoir quels sont les facteurs les plus déterminants. Toutefois, la "règle des pentes" (HUET, 1954) accorde à la pente un rôle prépondérant pour expliquer les discontinuités observées dans l'organisation longitudinale du peuplement.

3 - Lorsqu'un bassin fluvial est grand, il peut étendre son réseau sur différents espaces où les conditions géologiques, climatiques et écologiques sont différentes, indépendamment du gradient longitudinal. En réponse à ces variations, certaines espèces apparaissent ou disparaissent, et les proportions des espèces communes varient.

Cet article a pour objectif de déterminer les variations du peuplement liées, d'une part, à l'organisation longitudinale du milieu en tenant compte plus particulièrement des effets de la pente et, d'autre part, à la régionalisation des distributions.

\section{MATÉRIEL ET MÉTHODES}

\subsection{Bassin du Rhône}

De surface modeste à l'échelle du continent européen (96 $\left.000 \mathrm{~km}^{2}\right)$, le bassin du Rhône rassemble des milieux très contrastés, tant du point de vue du climat, que de celui de la géologie ou du relief (fig. 1).

La partie française du bassin ( $94 \%$ de sa surface) est scindée en six espaces distincts qui résument les quatre grandes tendances climatiques européennes : océanique, continentale, alpine et méditerranéenne. A l'est, se situent les Alpes, plus haut massif montagneux d'Europe occidentale, où règne un climat montagnard très prononcé. Le nord du bassin est occupé par la plaine de la Saône, formation alluviale de faible altitude sous climat océanique à tendance continentale, bordée à l'est par le Jura et au nord par les Vosges, massifs plus élevés où la tendance continentale s'accentue. Au sud du bassin, s'étend une zone méridionale sous climat méditerranéen bordée à l'ouest par les Cévennes, contreforts du Massif Central.

Avec un total de 56 espèces de poissons, plus deux formes de carpes, soit 58 taxons, le bassin du Rhône est le plus riche en espèces du pays (Tableau I).

La répartition des espèces natives a permis de délimiter trois régions ichtyogéographiques se rapportant aux trois plus importants sous-bassins (fig. 1). Ces régions identifient les structures biogéographiques du peuplement dominées par les processus historiques de colonisation-extinction (CHANGEUX et PONT, 1995). Pour savoir si ces régions pouvaient être étendues à la totalité du peuplement, la surface du bassin a 


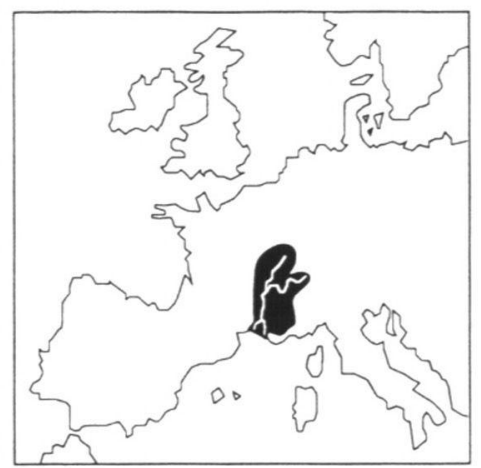

Régions ichtyogéographiques

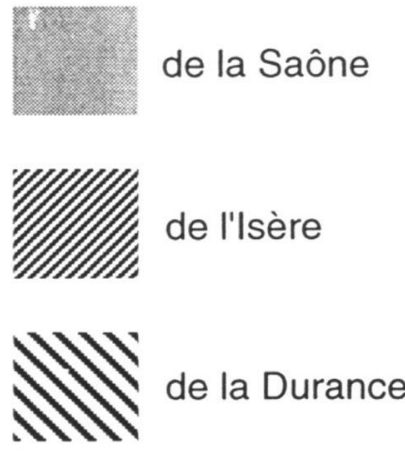

Petits sous-bassins

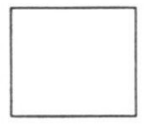

exclus des régions

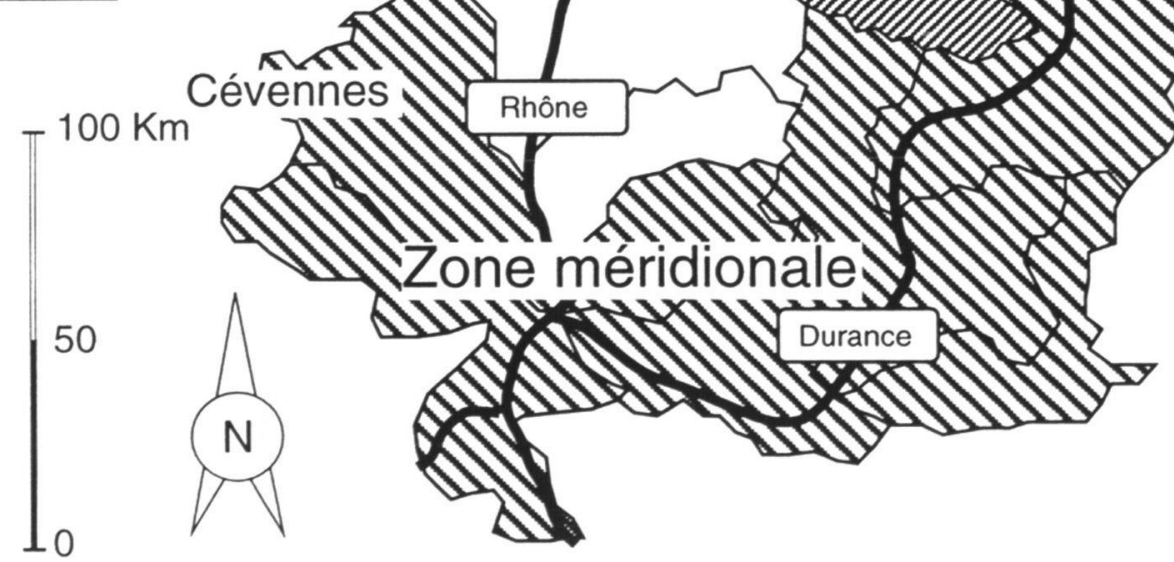

Figure 1: Le bassin français du Rhône avec les affluents principaux, les régions ichtyogéographiques et les limites des secteurs hydrologiques en traits fins.

Figure 1 : The French Rhone River basin with major tributaries and ichthyogeographic regions. The thin line shows the boundaries between hydrological sectors. 
Tableau I : Liste taxonomique des poissons du bassin du Rhône classés par famille, avec leur nom vernaculaire et latin ainsi que le code utilisé dans les autres figures.

Table I : Taxonomic list of the fishes of the Rhone basin ranked by families, with their French name, Latin name, and the code used in the other figures.

\begin{tabular}{|c|c|c|c|}
\hline Famille & Nom vernaculaire & Nom latin & Code \\
\hline \multirow[t]{2}{*}{ Petromyzontidae } & 1-lamproie de Planer & Lampetra planeri (Bloch, 1786) & LPP* \\
\hline & 2-lamproie marine & Petromyzon marinus (L., 1758) & LPM \\
\hline \multirow{3}{*}{$\begin{array}{l}\text { Anguillidae } \\
\text { Clupeidae }\end{array}$} & 3-anguille & Anguilla anguilla (L.,1758) & ANG \\
\hline & 4-alose feinte & Alosa fallax (Duhamel, 1772) & ALF \\
\hline & 5-grande alose & Alosa alosa (L.,1758) & ALA \\
\hline \multirow[t]{8}{*}{ Salmonidae } & 6-corégone & Coregonus sp (L.,1758) & $\mathrm{COR}^{*}$ \\
\hline & 7-cristivomer & Salvelinus namaycush (Walbaum,1794) & CRI \\
\hline & 8-huchon & Hucho hucho $(\mathrm{L} ., 1758)$ & HUC \\
\hline & 9-omble-chevalier & Salvelinus alpinus (L.,1758) & $\mathrm{OBL}^{*}$ \\
\hline & 10-saumon de fontaine & Salvelinus fontinalis (Mitchill, 1815) & SDF \\
\hline & 11-truite arc-en-ciel & Oncorhyncus mykiss (Walbaum, 1792) & TAC \\
\hline & 12-truite commune & Salmo trutta (L., 1758) & TRF $^{\star}$ \\
\hline & 13-ombre commun & Thymallus thymallus (L., 1758) & $\mathrm{OBR}^{*}$ \\
\hline Esocidae & 14-brochet & Esox lucius (L., 1758) & $\mathrm{BRO}^{*}$ \\
\hline \multirow[t]{24}{*}{ Cyprinidae } & 15-able de Hecke! & Leucaspius delinaetus (Heckel, 1843) & $\mathrm{ABH}$ \\
\hline & 16-ablette & Alburnus alburnus (L.,1758) & $A B L^{*}$ \\
\hline & 17-barbeau méridional & Barbus meridionalis (Risso, 1826) & BAM* $^{*}$ \\
\hline & 18-barbeau & Barbus barbus (L.,1758) & $\mathrm{BAF}^{\star}$ \\
\hline & 19-blageon & Leuciscus soufia (Risso, 1826) & $B L N^{*}$ \\
\hline & 20-bouvière & Rhodeus amarus (Bloch, 1785) & $\mathrm{BOU}^{*}$ \\
\hline & 21 -brème bordelière & Blicca bjoerkna $(\mathrm{L} ., 1758)$ & BRB $^{*}$ \\
\hline & 22-brème commune & Abramis brama (L., 1758) & BRE* $^{*}$ \\
\hline & 23-carassin & Carassius carassius (L., 1758) & CAS \\
\hline & 24-carpe commune & Cyprinus carpio (L.,1758) & $\mathrm{CCO}$ \\
\hline & 24-carpe cuir & Cyprinus carpio (L., 1758) & $\mathrm{CCU}$ \\
\hline & 24-carpe miroir & Cyprinus carpio (L., 1758) & CMI \\
\hline & 25-chevaine & Leuciscus cephalus (L., 1758) & $\mathrm{CHE}^{*}$ \\
\hline & 26-gardon & Rutilus rutilus (L.,1758) & GAR* \\
\hline & 27-goujon & Gobio gobio (L.,1758) & GOU* \\
\hline & 28-hotu & Chondrostoma nasus (L.,1758) & HOT \\
\hline & 29-psedorasbora & Pseudorasbora parva (Schlegel, 1842) & PSR \\
\hline & 30-poisson rouge & Carassius auratus (L.,1758) & CAA \\
\hline & 31-rotengle & Scardinius erythrophthalmus (L., 1758) & $\mathrm{ROT}^{*}$ \\
\hline & 32-spirlin & Alburnoides bipunctatus (Bloch, 1782) & $\mathrm{SPI}^{\star}$ \\
\hline & 33-tanche & Tinca tinca $(\mathrm{L} ., 1758)$ & TAN* $^{*}$ \\
\hline & 34-toxostome & Chondrostoma toxostoma (Vallot,1836) & TOX* \\
\hline & 35-vairon & Phoxinus phoxinus (L.,1758) & $\mathrm{VAl}^{*}$ \\
\hline & 36-vandoise & Leuciscus leuciscus (L., 1758) & VAN* $^{*}$ \\
\hline \multirow[t]{3}{*}{ Cobitidae } & 37-loche d'étang & Misgurnus fossilis (L., 1758) & LOE* $^{*}$ \\
\hline & 38 -loche de rivière & Cobitis taenia (L.,1758) & $\operatorname{LOR}^{*}$ \\
\hline & 39-loche franche & Nemacheilus barbatulus (L.,1758) & LOF $^{*}$ \\
\hline Siluridae & 40-silure glane & Silurus glanis (L., 1758) & SIL \\
\hline Ictaluridae & 41-poisson-chat & Ictalurus melas (Rafinesque, 1820 ) & $\mathrm{PCH}$ \\
\hline Gadidae & 42-lote de rivière & Lota lota $(L ., 1758)$ & LOT $^{\star}$ \\
\hline \multirow{2}{*}{ Mugilidae } & 43-mulet cabot & Mugil cephalus (L.,1758) & MUC \\
\hline & 44-mulet porc & Liza ramada (Risso, 1826) & MUP \\
\hline Poeciliidae & 45-gambusie & Gambusia affinis (Baird \& Girard,1853) & GAM \\
\hline \multirow[t]{2}{*}{ Gasterosteidae } & 46-épinochette & Pungitius pungitius (L.,1758) & $\mathrm{EPT}^{*}$ \\
\hline & 47-épinoche & Gasterosteus aculeatus (L., 1758) & EPI* \\
\hline Serranidae & 48-bar, loup & Dicentrarchus labrax (L.,1758) & LOU \\
\hline \multirow[t]{4}{*}{ Percidae } & 49-apron & Zingel asper $(\mathrm{L} ., 1758)$ & $\mathrm{APR}^{*}$ \\
\hline & 50-grémille & Gymnocephalus cernua (L., 1758) & GRE* \\
\hline & 51-perche & Perca fluviatilis (L., 1758) & PER* \\
\hline & 52-sandre & Stizostedion lucioperca $(\mathrm{L} ., 1758)$ & SAN \\
\hline \multirow[t]{2}{*}{ Centrarchidae } & 53-black bass & Micropterus salmoides (Lacépède,1802) & BBG \\
\hline & 54-perche soleil & Lepomis gibbosus (L.,1758) & PES \\
\hline Blenniidae & 55-blennie fluviatile & Blennius fluviatilis (Asso, 1801) & BLE* $^{*}$ \\
\hline Cottidae & 56-chabot & Cottus gobio (L.,1758) & $\mathrm{CHA}^{*}$ \\
\hline
\end{tabular}

\footnotetext{
* espèces natives
} 
été scindée en 21 secteurs hydrologiques de taille plus petite (fig. 1). Ce découpage, issu de la codification hydrologique utilisée par les Agences de l'Eau, respecte les limites de partage des eaux. Chaque secteur est repéré par une lettre indiquant le sous-bassin auquel il se rapporte suivi d'un chiffre qui précise son niveau de confluence.

\subsection{Source et sélection des données}

Une échelle aussi large que le bassin du Rhône nécessite l'emploi de données synthétiques simples pour décrire le milieu et le peuplement. Ces informations sont disponibles dans les "Schémas de vocation piscicole" (SOUCHON et TROCHERIE, 1990). Dans ces documents, les rivières sont découpées en tronçons unitaires, où les conditions de milieu et la composition du peuplement sont homogènes.

Le milieu est connu au travers de deux descripteurs synthétiques : la distance à la source et la pente du tronçon. Pour repérer les tronçons dans le gradient longitudinal, nous avons utilisé la distance à la source, répartie en sept classes $(<2$, de 2 à 4 , de 4 à 8 , de 8 à 16 , de 16 à 32 , de 32 à $64,>64 \mathrm{~km}$ ), déterminées de manière à faire le lien avec l'ordre de confluence de Strahler (LE FELLIC, 1991). Pour expliquer la majorité des discontinuités observées dans l'organisation longitudinale du peuplement, nous avons retenu la pente moyenne du tronçon, répartie en cinq classes ordonnées $(<1$, de 1 à 3 , de 3 à 10 , de 10 à $30,>30 \%$ o).

Le peuplement est connu, dans la majorité des cas, au travers d'une ou plusieurs pêches électriques. Sa composition a été retenue en présence(1)-absence(0) de taxon.

Nous nous sommes efforcés d'étudier plus particulièrement les tronçons des cours supérieurs et médians, où les effets des régions et du gradient longitudinal étaient susceptibles d'être les plus déterminants. Nous avons donc écarté les tronçons situés audelà de $500 \mathrm{~km}$ de distance à la source et limité le nombre de petits affluents au peuplement pauvre et redondant (CHANGEUX, 1994).

\subsection{Traitement des données}

Les données ont été informatisées puis analysées au moyen de la programmathèque ADE (DOLÉDEC et CHESSEL, 1993).

La présence d'un gradient dans le tableau faunistique nous a conduits à choisir la métrique du $\mathrm{Chi}^{2}$ utilisée dans l'Analyse Factorielle des Correspondances (AFC). Cette distance est particulièrement sensible aux taxons rares. C'est pourquoi nous avons systématiquement éliminé les taxons présents dans moins de $1 \%$ des tronçons considérés.

Compte tenu des restrictions précédentes, les données faunistiques se présentent sous la forme d'un tableau de 0 et de 1 avec 1202 tronçons de cours d'eau en ligne et 38 taxons en colonne. Le plan d'observation croisant pente et classes de distances à la source (CIDS) est complet (le Tableau II est sans cases vides). II permet d'appréhender l'action de ces deux descripteurs suivant un modèle additif, ou bien avec interaction.

Par ailleurs, les tronçons se répartissent entre les secteurs suivant leurs surfaces, avec une densité régulière de un tronçon pour $74,8 \mathrm{~km}^{2}$. Ce découpage, assez large, permet d'avoir un nombre non négligeable de tronçons par secteur (18 à 138). En outre, il isole plus clairement les grandes tendances régionales du peuplement. Un découpage plus fin comprend inévitablement des effets liés aux variations longitudinales d'autres facteurs locaux que la pente. Avec le risque de trouver des tendances régionales qui suivent le gradient longitudinal !

Chacun des 1202 tronçons est donc repéré par sa position géographique dans le bassin (appartenance à un des 21 secteurs) et sa position dans le gradient longitudinal en tenant compte de la pente (appartenance à une des 35 combinaisons de pente $\times$ CIDS). L'effet du positionnement des tronçons sur le peuplement a été mesuré en pourcentage d'inertie expliquée par rapport à l'inertie initiale du tableau, puis testé par permutation (POIZAT, 1993). Les variations ont été visualisées par des plans factoriels et les groupes ont été obtenus par classification ascendante hiérarchique sur les coordonnées factorielles en utilisant l'algorithme du lien moyen proposé par le logiciel MacDendro (THIOULOUSE, 1991). 
Tableau II : Plan d'observation, en nombre de sections par combinaison de classes de pente et de distance à la source.

Table II : Number of sections studied according to slope (5 classes) and distance from the source (7 classes).

\begin{tabular}{|c|c|c|c|c|c|c|c|}
\hline \multirow[b]{3}{*}{$n^{\circ}$} & \multirow{3}{*}{$\begin{array}{l}\text { Ordre de } \\
\text { confluence }\end{array}$} & \multirow{3}{*}{$\begin{array}{c}\text { Classe de Distance } \\
\text { à la Source (DS) }\end{array}$} & \multicolumn{5}{|c|}{$\mathrm{n}^{\circ}$ de Classe de Pente $(\mathrm{P})$} \\
\hline & & & 1 & 2 & 3 & 4 & 5 \\
\hline & & & $P \leq 1 \%$ & $1 \% \circ<P \leq 3 \%$ & $3 \%<<\leq 10 \%$ & $10 \% \circ<P \leq 30 \%$ & $P>30 \%$ \\
\hline 1 & $1-2$ & $D S \leq 2 \mathrm{~km}$ & 3 & 4 & 10 & 10 & 30 \\
\hline 2 & 3 & $2<\mathrm{DS} \leq 4 \mathrm{~km}$ & 9 & 15 & 30 & 31 & 42 \\
\hline 3 & 3 & $4<\mathrm{DS} \leq 8 \mathrm{~km}$ & 15 & 39 & 62 & 85 & 50 \\
\hline 4 & 4 & $8<\mathrm{DS} \leq 16 \mathrm{~km}$ & 8 & 51 & 56 & 88 & 27 \\
\hline 5 & 5 & $16<\mathrm{DS} \leq 32 \mathrm{~km}$ & 19 & 52 & 94 & 77 & 20 \\
\hline 6 & 6 & $32<D S \leq 64 \mathrm{~km}$ & 14 & 54 & 74 & 10 & 5 \\
\hline 7 & 7 & DS>64 km & 46 & 43 & 21 & 6 & 2 \\
\hline
\end{tabular}

\section{RÉSULTATS}

\subsection{Effets de la pente et de la distance à la source}

La décomposition de l'inertie du tableau faunistique, selon la pente et les CIDS (Tableau III), montre que la pente explique mieux les variations du peuplement que les CIDS $(8,6 \%$ de l'inertie expliquée contre $5,1 \%)$, malgré un nombre de modalités plus faible que les CIDS (5 contre 7 ).

La part de l'inertie due à l'effet d'interaction entre la pente et les CIDS (notée CIDS. Pente dans le Tableau III) s'élève à $2,5 \%$. Ce pourcentage est important puisqu'il est comparable à la part d'inertie expliquée par les CIDS sachant la pente $(2,3 \%)$. C'est pourquoi le modèle avec interaction porte à $13,4 \%$ la part d'inertie totale expliquée, ce qui est statistiquement significatif $(n=200$ permutations; $p+1,96 \sigma p \leq 0,02)$.

La part d'inertie expliquée par les 35 combinaisons pente $\times$ CIDS est visualisée par une AFC inter-combinaisons qui optimise la dispersion des peuplements moyens par combinaison (fig. 2). Le premier axe est particulièrement fort (fig. 2A). Le plan 1-2 s'organise suivant un arc (effet Guttman) en raison de l'importance de la structure en gradient du peuplement.

La répartition des taxons (fig. 2B) se fait suivant l'organisation classique des peuplements européens qui ordonne les taxons des plus rhéophiles, à droite, aux plus limnophiles, à gauche. Nous avons comparé nos résultats avec l'ordination simple, obtenue sur le peuplement du Doubs, affluent de la Saône (VERNEAUX, 1973, cf. p. 143). Les différences entre les rangs des taxons communs aux deux analyses sur l'axe 1 ne sont pas significatives (Test de Wilcoxon $Z=-0,29 ; N=27 ; p=0,77$ ). Chaque taxon est situé au même rang dans les deux ordinations (coefficient de corrélation de Spearman, $Z=3,6$; $\mathrm{N}=27 ; \mathrm{p}=0,0003$ ). On distingue la zone à truite commune, à ombre, à barbeau fluviatile et à brème commune. En amont de la zone à truite, le saumon de fontaine s'individualise. Dans la zone à brème, les formes de carpes cuir et miroir se positionnent plus en aval que la carpe commune.

La hiérarchie de l'action de la pente et des CIDS est illustrée par le plan de leurs combinaisons opposant, d'abord, les pentes faibles, à gauche, aux pentes fortes, à droite, puis, au sein de chaque groupe de pente, ordonnant les CIDS (fig. 2C). Le groupe de combinaisons à forte pente (entouré sur la fig. 2C) s'individualise clairement par rapport aux autres bien que la combinaison pente 5 x CIDS 6 (encadrée sur la fig. 2C) reste en position excentrée. Mis à part ce groupe particulier, où les CIDS n'ont aucun effet, les CIDS sont à peu près ordonnées en sens inverse à l'intérieur de chaque classe de pente (flèches noires de la fig. 2C). 
Tableau III : Décomposition de l'inertie du tableau faunistique d'AFC isolant l'effet de la Pente et des Classes de Distance à la Source (CIDS) suivant un modèle avec ou sans interaction.

Table III : Inertia decomposition of the Correspondence Analysis array showing the effects of slope (pente) and distance from the source (CIDS) following models with or without interaction.

\begin{tabular}{lcc}
\hline & Inertie (I) & Rapport (\%) \\
\cline { 2 - 3 } Pente (5 modalités) & 0,331 & 8,6 \\
CIDS sachant la Pente & 0,089 & 2,3 \\
interaction CIDS. Pente & 0,097 & 2,5 \\
Résidu & 3,344 & 86,6 \\
\hline Total & 3,861 & 100,0 \\
CIDS (7 modalités) & 0,196 & 5,1 \\
Pente sachant la CIDS & 0,224 & 5,8 \\
interaction CIDS. Pente & 0,097 & 2,5 \\
Résidu & 3,344 & 86,6 \\
\hline Total & 3,861 & 100,0 \\
Modèle additif & & \\
\hline Pente + CIDS & & 10,9 \\
Résidu & 0,420 & 89,1 \\
\hline Total & 3,441 & 100,0 \\
Modèle avec interaction & 3,861 & \\
combinaisons Pente x CIDS & & 13,4 \\
Résidu & & 86,6 \\
\hline Total & 0,517 & 100,0 \\
\hline
\end{tabular}

Ce schéma semble prévaloir sur l'ensemble du bassin. Les principaux écarts sont à mettre en relation avec des discontinuités du gradient propres à chaque cours d'eau, ou résultant de l'aménagement des rivières. Par exemple, le positionnement de la combinaison pente $5 \times$ CIDS 6 est lié à la présence d'un barrage (en amont d'un tronçon du Guiers (dépt. Isère)). La retenue agit comme un réservoir de poissons limnophiles qui, en diffusant vers l'aval, déplacent la combinaison vers la gauche du plan.

\subsection{Effets des secteurs hydrologiques}

La décomposition de l'inertie (Tableau IV) montre que, malgré un nombre de modalités plus faible (21 contre 35), la distinction des secteurs hydrologiques seule est à peu près aussi efficace pour expliquer les variations de peuplement que les combinaisons de pente $\times$ CIDS (11,5\% de l'inertie expliquée contre $13,4 \%)$.

Associés aux distinctions de pente et de CIDS, les effets des secteurs portent à $21 \%$ l'inertie totale expliquée. Les différences entre secteurs hydrologiques pouvant résulter de configurations différentes de pente et de CIDS, nous avons éliminé ces effets en retirant au peuplement observé le peuplement moyen de la combinaison à laquelle il se rapporte. Les secteurs, sachant les combinaisons pente $\times$ CIDS, expliquent encore $7,7 \%$ de l'inertie totale, ce qui est statistiquement significatif $(n=200$ permutations ; $p+1,96 \sigma p \leq 0,02$ ). 

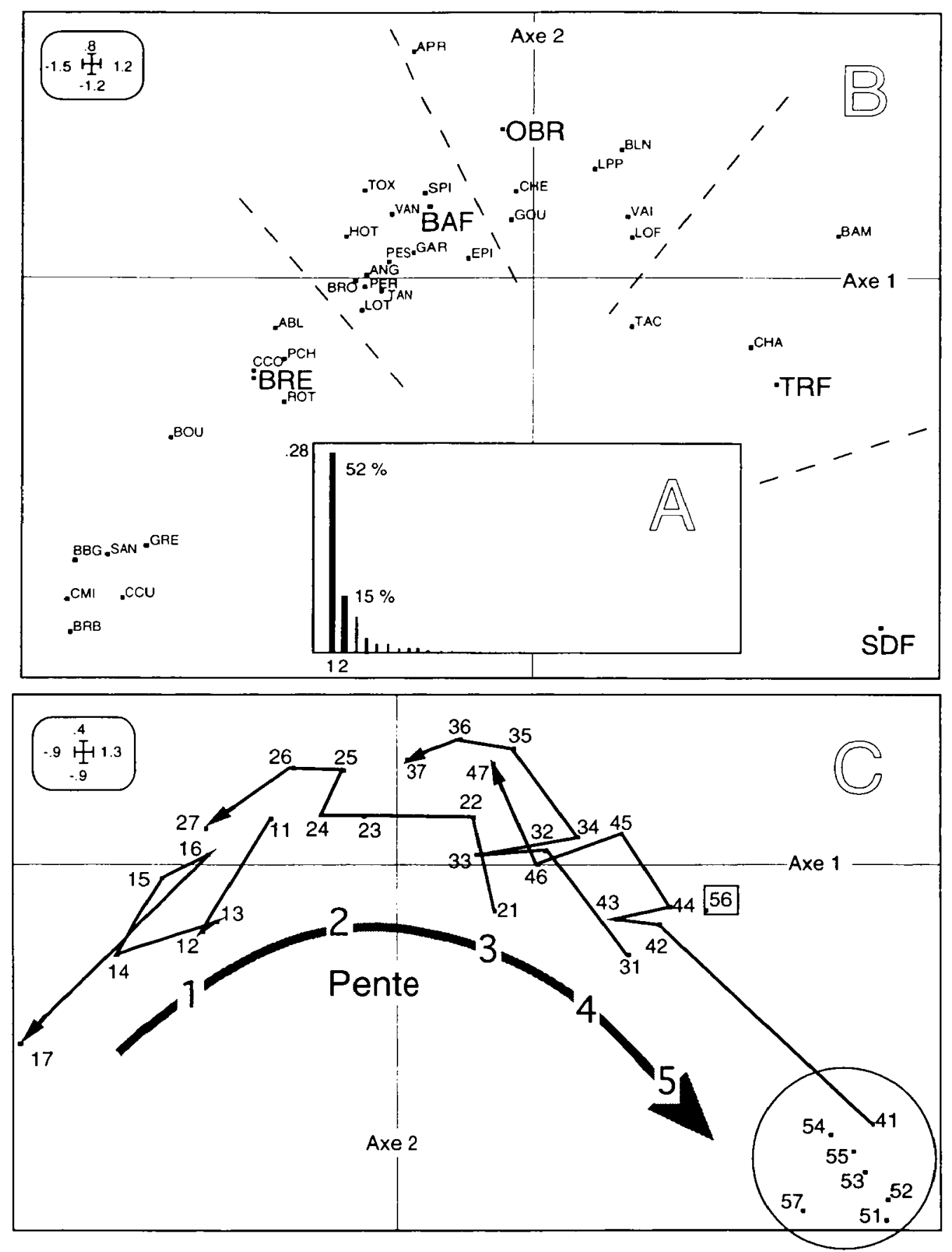

Figure 2 : AFC inter-combinaisons Pente $x$ Classes de Distance à la Source (CIDS). A : graphe des valeurs propres. B : plan 1-2 des taxons codés selon le Tableau I (les codes espèces marquantes sont agrandis). $C$ : plan 1-2 des combinaisons avec les flèches joignant les combinaisons de même classe de pente (premier chiffre) dans l'ordre des CIDS (deuxième chiffre) croissantes.

Figure 2 : Correspondence Analysis between combinations of slope (pente) and distance from the source (CIDS). A : Graph of eigen values. B : Taxa factorial map on the first two axes (codes are given Table I, those of the most significant species are enlarged). C : Combination factorial map on the same axis, with the arrows joining combinations of the same slope class (first figure) sorted by increasing distance from the source (second figure). 
Tableau IV : Décomposition de l'inertie du tableau faunistique d'AFC selon l'effet du Secteur Hydrologique (SH) seul, puis en prenant en compte l'effet de la Pente et des Classes de Distance à la source (CIDS).

Table IV : Inertia decomposition of the Correspondence Analysis array according to the hydrological sectors (SH) only, then after taking into account the slope (pente) and the distance from the source (CIDS).

\begin{tabular}{lcc}
\hline & Inertie & Rapport (\%) \\
\hline SH (21 modalités) & 0,445 & 11,5 \\
Résidu & 3,416 & 88,5 \\
\hline Total du tableau & 3,861 & 100,0 \\
Combinaisons Pente x CIDS (35 modalités) & 0,517 & 13,4 \\
SH sachant la combinaison Pente x CIDS & 0,296 & 7,7 \\
\hline Total expliqué & 0,813 & 21,1 \\
Résidu & 3,048 & 78,9 \\
\hline Total du tableau & 3,861 & 100,0 \\
\hline
\end{tabular}

Cette part d'inertie a été visualisée par une AFC inter-secteurs qui optimise la dispersion des peuplements moyens par secteur, une fois retiré l'effet du gradient de pente et de distance à la source. Le graphe des valeurs propres (fig. $3 \mathrm{~A}$ ) montre que le tableau a une structure simple qui peut se résumer sur les deux premiers axes.

Dans le plan des taxons (fig. 3B), l'ordination amont-aval du peuplement est rompue au bénéfice des espèces aux distributions plus régionalisées (barbeau méridional, saumon de fontaine, ombre, truite arc-en-ciel, black-bass, anguille).

Dans le plan des secteurs (fig. $3 C$ et $3 D$ ), le premier axe oppose les secteurs montagneux des Alpes ou Périalpins à la Zone méridionale. La classification hiérarchique scinde les secteurs montagneux en deux groupes : les Alpes internes avec les secteurs amont du bassin de l'Isère (W1, WO) et les massifs élevés qui rassemblent les Préalpes calcaires (W2, VO), le Haut-Jura (V2) et les Hautes-Alpes du sud (X0). Les zones méridionales concernent la partie aval du bassin de la Durance $(X 2, X 3)$ et les affluents du Rhône situés au sud de la confluence avec l'Isère (V4, V5, V6, V7). Enfin, le second axe oppose les secteurs des Alpes centrales aux secteurs nordiques des plaines de la Saône et du Rhône (U3, U4, V3) associés aux zones de moyennes montagnes que sont les Vosges $(\cup 0)$, le Jura $(U 2, U 1)$ et les Préalpes de l'ouest $\left(V_{1}, W 3\right)$. Bien qu'il soit situé dans la partie sud du bassin, le secteur des Alpes du sud (X1) est également rattaché à ce groupe mais se rapproche beaucoup des zones méridionales.

\section{DISCUSSION ET CONCLUSION}

L'emploi de descripteurs synthétiques tels que la pente, la distance à la source ou les secteurs hydrologiques, pour étudier la relation habitat-poisson à l'échelle d'un bassin est particulièrement efficace car ces trois variables suffisent à expliquer $21 \%$ des variations observées dans le peuplement des cours supérieurs et médians. Toutefois, l'interprétation des résultats obtenus est difficile par manque d'étude faisant le lien entre ces descripteurs et les facteurs agissant aux échelles inférieures où se situent les poissons.

\subsection{Structure globale du peuplement rhodanien}

Le rôle primordial de la pente sur la structure globale du peuplement confirme sur des critères statistiques la "règle des pentes". La géologie particulière et l'abondance des aménagements, propre au bassin rhodanien, sont responsables d'une multitude d'écarts 


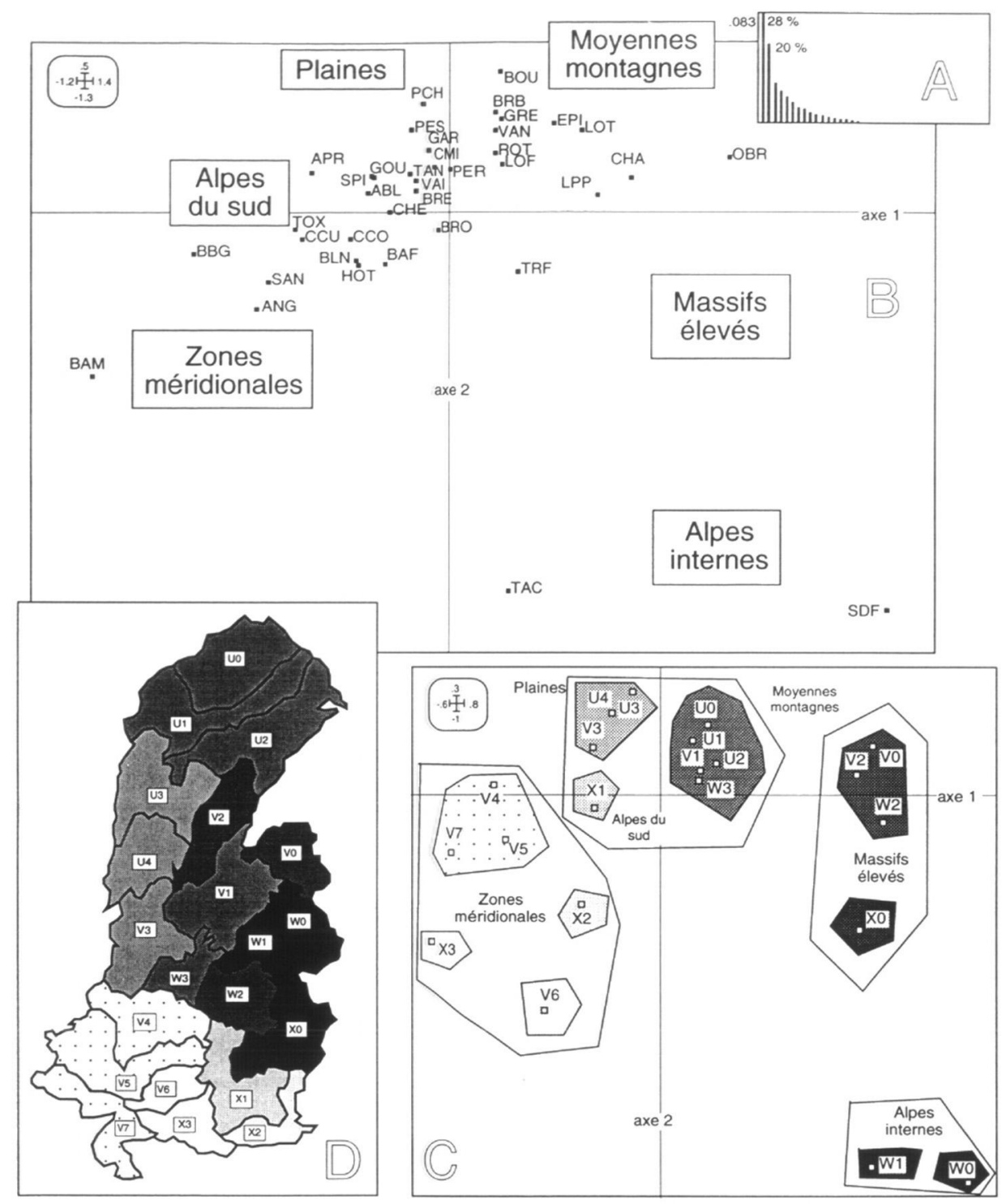

Figure 3 : AFC inter-secteurs hydrologiques après retrait de l'effet de la Pente et des Classes de Distance à la Source (CIDS). A : graphe des valeurs propres. B : plan 1-2 des taxons codés selon le Tableau I. C : plan 1-2 des secteurs hydrologiques, identifiés par une lettre, indiquant le bassin versant auquel ils appartiennent (U : Saône, V : Rhône, $W$ : Isère, $X$ : Durance), et un chiffre, qui les ordonne d'amont en aval suivant leur niveau de confluence. $D$ : cartographie des tendances régionales après classification ascendante hiérarchique utilisant le lien moyen (deux niveaux de hiérarchie sont indiqués, les trames sont attribuées en fonction de la position sur l'axe 1 des groupes les plus petits).

Figure 3 : Correspondence Analysis between hydrological sectors after elimination of slope and distance from the source effects. A : Graph of eigen values. B : Taxa factorial map on the first two axes (codes are given Table I). C : Hydrological sectors, coded by a letter showing the subcatchment to which they belong ( $U$ : Saone, $V:$ Rhone, $W$ : Isère, $X$ : Durance) and a figure which sorts them from upstream to downstream according to the level of their confluence. D : Geographical display of regional tendencies after average cluster analysis (two levels of partition are indicated, and shadings are determined by the position of the smaller groups on the first axis). 
au profil d'équilibre qui introduisent des discontinuités dans la dimension longitudinale. Ceci explique l'influence relativement faible de la distance à la source par rapport à la pente dans ce bassin.

L'ordination des poissons suivant leur degré de rhéophilie (fig. 2) montre que la pente intervient essentiellement au travers de la vitesse d'écoulement de l'eau. La progression du nombre d'espèces avec la distance à la source reste un trait commun, bien connu, des cours supérieurs et médians européens.

L'antagonisme entre la pente et la distance à la source peut résulter de la corrélation négative entre ces deux paramètres qui persiste à l'intérieur des classes qui restent relativement larges. Pour une classe de pente donnée, les tronçons les plus éloignés de la source seraient ceux qui présentent la pente la plus faible et inversement.

Enfin, l'interaction pente-distance à la source est illustrée par les tronçons de la classe de pente 5 sur laquelle les variations de distance à la source n'ont aucun effet. Elle se retrouve également, dans une moindre mesure, au niveau des variations d'ordination observées entre les classes de pente inférieures. Ces variations traduisent l'intensité des modulations du gradient longitudinal par d'autres paramètres que la pente comme, par exemple, des tendances régionales.

\subsection{Identification des tendances régionales}

Avec les secteurs hydrologiques, nous avons isolé des tendances régionales du peuplement, qui n'étaient pas distinguées dans le gradient global de pente et de distance à la source. Elles concernent des espèces à distribution régionalisée qui, contrairement à celles utilisées pour déterminer les régions ichtyogéographiques, sont parfois introduites.

Les tendances régionales mises en évidence se distinguent des régions ichtyogéographiques. Elles sont relativement indépendantes de la répartition en sousbassin (e.g. les secteurs relevant du bassin de la Durance se répartissent entre trois groupes) et n'ont pas toujours de continuité géographique. Elles répondent surtout à des traits climatiques contemporains. La figure 3D permet d'identifier trois influences majeures : l'influence montagnarde, qui concerne les Alpes internes et les massifs élevés, l'influence méditerranéenne qui s'exprime dans les zones méridionales et s'oppose à l'influence océanique à tendance continentale, qui règne dans la plaine de la Saône, dans les Vosges et le Jura.

Le peuplement des Alpes internes est marqué par des espèces introduites : le saumon de fontaine et la truite arc-en-ciel ; celui des plaines et des zones de moyenne montagne par la bouvière, l'épinoche, la lote, la grémille, le poisson-chat et la brème bordelière ; celui de la zone méridionale et les Alpes du sud par le barbeau méridional, l'anguille, le black-bass, le sandre, le toxostome, la carpe cuir, l'apron, la carpe commune, et le blageon; enfin, celui des massifs élevés par la truite, le chabot, l'ombre et la lamproie de Planer.

\subsection{Composantes des distinctions régionales}

Les particularités faunistiques des cours d'eau nordiques et méridionaux sont connues depuis longtemps (DORIER, 1957 ; ILLIES et BOTOSANEANU, 1963). La température est généralement retenue comme le facteur déterminant dans ces variations de répartition en liaison avec la latitude qui intègre les variations temporelles du climat.

Dans le bassin du Rhône, il faut considérer également l'effet de l'altitude qui varie beaucoup d'un secteur à l'autre. Ce paramètre est aussi fortement corrélé avec la température et intègre de nombreuses autres variables climatiques dans le temps (intensité et régime des précipitations, enneigement). Pour une région ichtyogéographique donnée, tant que les variations d'altitude ne sont pas trop importantes, l'élimination des effets de la distance à la source et de la pente suffit à retirer toute structure longitudinale (e.g. les secteurs de la Saône fig. $3 \mathrm{C}$ ). En revanche, si on s'adresse à une région ichtyogéographique où les variations d'altitudes intersectorielles sont importantes, alors ce paramètre intervient en 
modulant les effets de la distance à la source et de la pente. Par exemple, dans la région de l'Isère, elle fait apparaître plusieurs tendances importantes en abaissant la température, en introduisant dans le régime hydrologique une composante glaciaire (débit maximum en été) et en favorisant les débits importants.

Tous ces paramètres essentiels pour comprendre la relation habitat-poisson, à l'échelle d'un grand bassin fluvial, traduisent des variations du climat. Ainsi, comme pour les peuplements végétaux, le climat est responsable de nombreuses distinctions régionales contemporaines et surimpose son action sur les héritages biogéographiques déjà révélés par les régions ichtyogéographiques.

\section{REMERCIEMENTS}

Je tiens à remercier tous les membres du Laboratoire d'Écologie des Systèmes Fluviaux, en particulier Didier PONT qui est à l'origine de ce travail et Gilles POIZAT qui a relu une première version du manuscrit. Cette recherche a été financée par une bourse de docteur ingénieur du Centre National de la Recherche Scientifique. Elle est intégrée au Programme Interdisciplinaire de Recherche sur l'Environnement intitulé "Signification des échelles spatiotemporelles dans la variabilité des peuplements piscicoles des grands cours d'eau».

\section{BIBLIOGRAPHIE}

CHANGEUX T., 1994. Structure des peuplements de poissons à l'échelle du bassin rhodanien. Approche régionale et organisation longitudinale. Exploitation des captures par pêche aux engins. Thèse de doctorat, Univ. Cl. Bernard, Lyon 1, $242 \mathrm{p}$.

CHANGEUX T., PONT D., 1995. Ichthyogeographic regions and watershed size in the French river Rhône network. Hydrobiologia, 300-301, 355-363.

DOLÉDEC S., CHESSEL D., 1993. ADE Software: Multivariate Analyses and Graphical Display for Environmental Data, Version 3.6. URA CNRS 1451, Univ. Cl. Bernard Lyon 1.

DORIER A., 1957. Répartition du Barbeau méridional dans le sud-est de la France. Trav. Lab. Hydrobiol. Pisc. Grenoble, 48-49, 141-149.

HUET M., 1954. Biologie, profils en long et en travers des eaux courantes. Bull. Fr. Piscic., 175, 41-53.

ILLIES J., BOTOSANEANU L., 1963. Problèmes et méthodes de la classification et de la zonation écologique des eaux courantes, considérés surtout du point de vue faunistique. Mitt. Int. Verein. Theor. Angew. Limnol., 12, 1-57.

LE FELLIC A., 1991. Analyse quantitative des systèmes hydro-graphiques du bassin Rhône-Méditerrannée-Corse et applications en termes de gestion. Rapport de DEA, Univ. J. Moulin, Lyon 3, 109 p.

POIZAT G., 1993. Echelle d'observation et variabilité des abondances de juvéniles de poissons dans un secteur aval du Rhône. Thèse de doctorat, Univ. Cl. Bernard Lyon 1, $217 \mathrm{p}$.

SOUCHON Y., TROCHERIE F., 1990. Technical aspects of French legislation dealing with the freshwater fishery (June 1984): 'Fisheries orientation schemes' and 'Fishery resources management plans' in Van Densen W.L.T., Steinmetz B. and Hughes R.H., Management of freshwater fisheries, 190-214, Pudoc, Wageningen, The Netherlands.

THIOULOUSE J., 1991. MacDendro, logiciel de classification hiérarchique, version 3.02, URA CNRS 243, Université Cl. Bernard Lyon 1.

VERNEAUX J., 1973. Cours d'eau de Franche-Comté (Massif du Jura). Recherches écologiques sur le réseau hydrographique du Doubs. Essai de biotypologie. Thèse de doctorat, Univ. des Sciences et Techniques de Besançon, $257 \mathrm{p}$. 